\title{
Herschel/PACS photometry of transiting-planet host stars with candidate warm debris disks ${ }^{\star}$
}

\author{
Bruno Merín ${ }^{1}$, David R. Ardila², Álvaro Ribas ${ }^{3,1}$, Hervé Bouy ${ }^{3}$, Geoffrey Bryden ${ }^{4}$, \\ Karl Stapelfeldt ${ }^{5}$, and Deborah Padgett ${ }^{6,7}$
}

\author{
${ }^{1}$ European Space Astronomy Centre (ESA), PO Box 78, 28691 Villanueva de la Cañada, Spain \\ e-mail: bmerin@sciops.esa.int \\ 2 NASA Herschel Science Center, California Institute of Technology, Mail Code 100-22, Pasadena, CA 91125, USA \\ 3 Centro de Astrobiología, INTA-CSIC, PO Box - Apdo. de correos 78, Villanueva de la Cañada Madrid 28691, Spain \\ 4 Jet Propulsion Laboratory, California Institute of Technology, Pasadena, CA 91109, USA \\ 5 NASA Goddard Space Flight Center, Exoplanets and Stellar Astrophysics, Code 667, Greenbelt, MD 20771, USA \\ 6 Spitzer Science Center, California Institute of Technology, Pasadena, CA 91125, USA \\ 7 Current address: NASA Goddard Space Flight Center, Code 667, Greenbelt, MD 20771, USA
}

Received 31 October 2013 / Accepted 2 August 2014

\section{ABSTRACT}

\begin{abstract}
Dust in debris disks is produced by colliding or evaporating planetesimals, which are remnants of the planet formation process. Warm dust disks, known by their emission at $\leq 24 \mu \mathrm{m}$, are rare ( $4 \%$ of FGK main sequence stars) and especially interesting because they trace material in the region likely to host terrestrial planets, where the dust has a very short dynamical lifetime. Statistical analyses of the source counts of excesses as found with the mid-IR Wide Field Infrared Survey Explorer (WISE) suggest that warm-dust candidates found for the Kepler transiting-planet host-star candidates can be explained by extragalactic or galactic background emission aligned by chance with the target stars. These statistical analyses do not exclude the possibility that a given WISE excess could be due to a transient dust population associated with the target. Here we report Herschel/PACS 100 and 160 micron follow-up observations of a sample of Kepler and non-Kepler transiting-planet candidates' host stars, with candidate WISE warm debris disks, aimed at detecting a possible cold debris disk in any one of them. No clear detections were found in any one of the objects at either wavelength. Our upper limits confirm that most objects in the sample do not have a massive debris disk like that in $\beta$ Pic. We also show that the planet-hosting star WASP-33 does not have a debris disk comparable to the one around $\eta$ Crv. Although the data cannot be used to rule out rare warm disks around the Kepler planet-hosting candidates, the lack of detections and the characteristics of neighboring emission found at far-IR wavelengths support an earlier result suggesting that most of the WISE-selected IR excesses around Kepler candidate host stars are likely due to either chance alignment with background IR-bright galaxies and/or to interstellar emission.
\end{abstract}

Key words. planetary systems - planets and satellites: dynamical evolution and stability

\section{Introduction}

The existence of debris disks around stars has been known since the first far-infrared (far-IR) observations from space were carried out. The first debris disk was found around Vega by the Infrared Astronomical Satellite (IRAS; Aumann et al. 1984), but hundreds of stars were revealed to host them soon after (e.g., Oudmaijer et al. 1992; Mannings \& Barlow 1998). These disks are thought to be analogs of the Kuiper belt (Moro-Martín 2008), comprised of planetesimals, which are the remnant of the planet formation process. Their detection is due to the presence of dust in these belts produced by collisions among or evaporation of planetesimals (e.g., Wyatt 2008). Typical debris disks have estimated radii of some hundred $\mathrm{AU}$ and brightness temperatures around $\sim 50 \mathrm{~K}$. This results in a peak in their spectral energy distribution (SED) around 70-100 $\mu \mathrm{m}$. They are a normal feature in the universe, with an estimated frequency of $\sim 16$ to $20 \%$ around main sequence FGK stars, from Spitzer and Herschel studies, respectively (Trilling et al. 2008; Eiroa et al. 2013).

\footnotetext{
* Herschel is an ESA space observatory with science instruments provided by European-led Principal Investigator consortia and with important participation from NASA.
}

A fraction of debris disks emit significantly at shorter wavelengths, suggesting higher dust temperatures and hence smaller separations of the dust from the host star. Compared to the previously mentioned frequency of debris disks in main sequence FGK stars, only $\sim 4 \%$ of solar-type stars were found to show excesses at $24 \mu \mathrm{m}$ by the Spitzer Space Telescope (Trilling et al. 2008). In most of the cases, this is produced by the Wien edge of longer wavelength dust emission (e.g., a highly massive debris disk), although some stars have been proved to harbor legitimate warm inner debris disks (Siegler et al. 2007; Lawler et al. 2009).

There is no single explanation for these kinds of warm excesses for objects older than $\sim 100$ Myrs. Melis et al. (2010) shows that for these ages, it is still possible to find strong mid-IR excesses due to the destruction of planetary embryos, resulting in a dusty environment in the inner regions of the host star. For older systems warm dust close to the star cannot be continuously replenished. For example, the steady state asteroid-belt planetesimal grinding model by Wyatt et al. (2007) is not capable of explaining these warm excesses. Therefore, they are likely to be produced in transient events such as planetesimals collisions owing to gravitational instabilities that cause bodies in the outer disk to be pushed into their asteroid 
belt (Absil et al. 2006; Wyatt 2008). Another possible explanation involves perturbation of close-in planets in the asteroid belt (see Wyatt 2008).

In a recent study, (Ribas et al. 2012, hereafter R12), we searched for warm IR excesses around the 997 stars released as candidate planetary host stars by the Kepler project in February 2011. See also Lawler \& Gladman (2012) for a similar work on the same topic. The sample of stars observed by Kepler is comprised of over 150000 stars determined by the Kepler Input Catalog (KIC, Latham et al. 2005; Batalha et al. 2010), harboring mostly main sequence $G$ type field dwarfs. Morton \& Johnson (2011) suggest that 90-95\% of these candidates are real planetary systems, although the fraction of false positives and contamination is still under a strong debate (see, e.g., Mann et al. 2012; Adams et al. 2013; Lillo-Box et al. 2014). This catalog was combined with the Wide-field Infrared Survey Explorer (WISE) Preliminary Release Catalog (PRC) ${ }^{1}$, which covered $\sim 57 \%$ of the sky and half of the Kepler field of view at $3.4,4.6,12$, and $22 \mu \mathrm{m}$ (hereafter $W 1, W 2, W 3$, and $W 4$ ). Using the $W 3$ and $W 4$ bands, R12 selected 13 objects in half the Kepler field as warm dust excess candidates.

On February 2012 the Kepler mission released a new sample of 2321 planetary systems candidates around 1790 stars $^{2}$. On March 2012, the WISE All-Sky Catalog (ASC) was released ${ }^{3}$, covering the complete Kepler field and providing an unprecedented opportunity to study the warm disks' incidence in an homogeneous sample and obtaining more significant statistical values.

Here we present an update of the work done in R12 using the new Kepler candidates and the WISE All-Sky Catalog, plus follow-up observations at 100 and $160 \mu \mathrm{m}$ of a refined sample done with the PACS instrument (Poglitsch et al. 2010) onboard the Herschel Space Observatory (Pilbratt et al. 2010). The aim of this work is to potentially detect a large IR excess at longer wavelengths indicating a large debris disk in one of the new candidate planet-hosting stars or, alternatively, to clarify the possible contamination of these mid-IR excesses by extra galactic sources, as recently proposed by Kennedy \& Wyatt (2012). Non-detections with Herschel would suggest a lack of a cold-component in the debris disk but cannot rule out the presence of a warm debris disk only emitting at mid-IR wavelengths. Recent evidence of multi-component debris disks (Su et al. 2013; Absil et al. 2013) indicates a potential for discovering the cold component of such disks around any of these candidates.

This work is organized as follows. Section 2 describes the definition of the new sample to be followed up with Herschel based on the latest Kepler sample of planetary system candidates, transiting planet systems, and the WISE ASC. Section 3 describes the Herschel/PACS observations of the sample and the data reduction. Section 3.1 shows the images, describes the flux measurements, and analyzes the resulting SEDs of the targets. The discussion and final conclusions are given in Sect. 4 .

\section{Candidate selection}

\subsection{Catalog matching}

We followed the same approach as R12, by searching the 1790 Kepler planetary systems' candidate stars and previously known transiting systems ${ }^{4}$ as described in Poddaný et al. (2010)

\footnotetext{
1 http://wise2.ipac.caltech.edu/docs/release/prelim/

2 http://archive.stsci.edu/kepler/planet_candidates. html

3 http://wise2 .ipac.caltech.edu/docs/release/allsky/

4 http://var2.astro.cz/ETD/index.php
}

for counterparts to the WISE catalog. We considered a search radius of $1^{\prime \prime}$ as a good agreement between the WISE astrometric accuracy $\left(\sim 0.2^{\prime \prime}\right.$, see the Explanatory Supplement to the WISE All-Sky Data Release) and a conservative cut in order not to lose potential candidates. A total of 1728 matches was obtained with this process.

The catalog was then cleaned in the following way. We required photometry from the 2 Micron All Sky Survey (2MASS, Skrutskie et al. 2006) with signal-to-noise ratio (S/N) $\geq 7$ and no problems with artifacts or halos. We also selected only those sources marked as uncontaminated by extragalactic sources in the catalog. Objects with magnitudes below the saturation limit in any WISE bands are rejected, as well as those classified as extended. As a last prerequisite, we selected only those objects with $S / N \geq 3$ at $W 3$ band in order to try to make sure that the detections are real. This whole process led to selecting 844 candidates.

\subsection{Target selection}

Following the procedure in R12, we defined the excess at 12 or $22 \mu \mathrm{m}$ as $\chi_{\lambda} \equiv\left(F_{\text {WISE }}-F_{\text {phot }}\right) / \sigma_{\text {tot }} \geq 2$, where $F_{\text {WISE }}$ is the detected, dereddened flux at $W 3$ or $W 4$ band, $F_{\text {phot }}$ is the corresponding synthetic flux value obtained from photospheric modeling, and $\sigma_{\text {tot }}$ represents the total uncertainty computed as $\sigma_{\text {tot }}=\sqrt{\sigma_{\text {obs }}^{2}+\sigma_{\text {cal }}^{2}}$, where $\sigma_{\text {obs }}$, the measurement uncertainty in the corresponding band, and $\sigma_{\text {cal }}$ the absolute calibration uncertainty of the WISE instrument (see the Explanatory Supplement to the WISE All-Sky Data Release). We selected those objects with $\chi_{\lambda}>2$ (corresponding to a $95 \%$ confidence level) at one or both $W 3$ and $W 4$ bands (when detected), and obtained 293 objects.

\subsection{WISE image inspection}

The WISE images of the remaining candidates were visually inspected in order to assure they are real detections. The study in R12 showed that a significant number of the sources in the WISE PRC suffered from photometric problems or were spurious detections. We followed the same approach, rejecting sources based on three criteria (see R12 for different examples):

- Artifacts: The source is contaminated by halos or extended emissions.

- Offsets: The centroid position of the emission peak of the source at the band where excess is found is different than at $W 1$ or $W 2$ bands.

- "Extended" point spread functions (PSFs) or no detections: The photocenter at the band where the excess is found does not appear as a single PSF. This may be because the source is extended, because additional sources are present nearby at the same flux level or because the source is faint enough that significant noise can contribute to the detection.

Table 1 lists the final 19 objects that passed all selection criteria above and their corresponding $\chi_{\lambda}$ values after the process above. All but one of the 19 selected sources show strong $12 \mu \mathrm{m}$ excess, suggesting that they are due to warm disks rather than the Wien tail of colder disks. The second column in the table gives the Kepler Object of Interest (KOI) identifications of the objects where available. A few objects that were observed by us with PACS were not identified by Kepler as KOIs at the time of our Herschel observations, but were observed as a control sample 
B. Merín et al.: Herschel/PACS photometry of transiting-planet host stars with candidate warm debris disks

Table 1. Final sample fulfilling all selection criteria, observing log and results from the Herschel/PACS follow-up observations.

\begin{tabular}{lllllllllc}
\hline \hline Object & Alt. name & RA (J2000) & Dec (J2000) & $\chi_{12}$ & $\chi_{22}$ & $\begin{array}{c}\text { Herschel } \text { obsids } \\
13422 . .\end{array}$ & $\begin{array}{c}F_{100 \mu \mathrm{m}} \\
(\mathrm{mJy})\end{array}$ & $\begin{array}{c}F_{16 \mu \mu \mathrm{m}} \\
(\mathrm{mJy})\end{array}$ & $\begin{array}{c}\text { Separation } \\
\left({ }^{\prime}\right)\end{array}$ \\
\hline CoRoT-10 & & $19: 24: 15.29$ & $+00: 44: 45.99$ & 4.12 & $\ldots$ & 49290,91 & $<5.8$ & $<23.8$ & 12.3 \\
HAT-P-28 & & $00: 52: 00.18$ & $+34: 43: 42.22$ & 2.67 & $\ldots$ & 59254,55 & $<3.1$ & $<5.8$ & 6.1 \\
WASP-33 & & $02: 26: 51.06$ & $+37: 33: 01.73$ & -0.14 & 2.11 & 49290,91 & $<2.8$ & $<5.0$ & 27.1 \\
KIC 2162635 & KOI-1032 & $19: 27: 54.62$ & $+37: 31: 57.00$ & 9.34 & 3.63 & 57407,08 & $<6.33$ & $<17.6$ & 28.1 \\
KIC 3835670 & KOI-149 & $19: 06: 31.22$ & $+38: 56: 44.23$ & 6.30 & $\ldots$ & 56956,57 & $<4.7$ & $<8.7$ & 3.7 \\
KIC 4478168 & KOI-626 & $19: 40: 46.41$ & $+39: 32: 23.00$ & 3.62 & $\ldots$ & 57409,10 & $<3.5$ & $<7.1$ & 25.0 \\
KIC 4918309 & KOI-1582 & $19: 20: 30.87$ & $+40: 01: 18.61$ & 3.77 & $\ldots$ & 59364,65 & $<2.7$ & $<8.7$ & 3.4 \\
KIC 7097965 & & $18: 57: 57.68$ & $+42: 38: 53.77$ & 2.65 & $\ldots$ & 56212,13 & $<2.7$ & $<7.5$ & 18.8 \\
KIC 8766650 & & $19: 46: 11.42$ & $+44: 56: 30.71$ & 7.73 & $\ldots$ & 53513,14 & $<4.0$ & $<8.8$ & 38.9 \\
KIC 8962094 & KOI-700 & $19: 39: 53.65$ & $+45: 12: 49.32$ & 7.73 & 2.94 & 57740,41 & $<8.8$ & $<24.9$ & 28.2 \\
KIC 9703198 & KOI-469 & $19: 14: 33.08$ & $+46: 25: 17.17$ & 4.56 & $\ldots$ & 57742,43 & $<3.5$ & $<7.9$ & 13.1 \\
KIC 9884104 & KOI-718 & $19: 14: 57.34$ & $+46: 45: 45.35$ & 3.12 & $\ldots$ & 59238,39 & $<2.4$ & $<15.4$ & 18.4 \\
KIC 9965439 & KOI-722 & $19: 49: 02.19$ & $+46: 50: 35.50$ & 2.71 & $\ldots$ & 59240,41 & $<3.6$ & $<7.3$ & 16.6 \\
KIC 10386922 & KOI-289 & $18: 51: 46.94$ & $+47: 34: 29.71$ & 2.46 & $\ldots$ & 57744,45 & $<3.2$ & $<8.8$ & 19.6 \\
KIC 10873260 & KOI-535 & $19: 45: 32.53$ & $+48: 14: 00.25$ & 5.24 & $\ldots$ & 59242,43 & $<5.3$ & $<14.0$ & 20.5 \\
KIC 11134879 & KOI-480 & $19: 21: 45.02$ & $+48: 47: 30.83$ & 3.54 & $\ldots$ & 56215,16 & $<8.3$ & $<11.7$ & 10.8 \\
KIC 11624249 & KOI-356 & $19: 50: 56.73$ & $+49: 38: 13.62$ & 5.04 & $\ldots$ & 59244,45 & $<6.5$ & $<15.2$ & 16.5 \\
KIC 11673802 & & $19: 49: 26.24$ & $+49: 47: 51.21$ & 5.95 & 3.70 & 57970,71 & $<4.2$ & $<20.8$ & 24.6 \\
KIC 11774991 & & $19: 49: 10.21$ & $+49: 58: 54.23$ & 3.15 & $\ldots$ & 57968,69 & $<3.1$ & $<8.1$ & 31.8 \\
\hline
\end{tabular}

Notes. ${ }^{(a)}$ Herschel reset of the Spacecraft Velocity Vector (corrected in HCSS v.10.1). ${ }^{(b)}$ WISE ASC flag w4flg indicates source confusion at $W 4$ band. ${ }^{(\dagger)}$ Separation between intended target position and the closest point source centroid as measured in the $100 \mu \mathrm{m}$ image.

Table 2. Updated $\chi_{12}$ and $\chi_{22}$ values for the previous sample from R12 using the WISE ASC, together with notes from the image inspection.

\begin{tabular}{llllll}
\hline \hline Object & RA $(\mathrm{J} 2000)$ & Dec $(\mathrm{J} 2000)$ & $\chi_{12}$ & $\chi_{22}$ & Notes from image inspection \\
\hline WASP-46 & $21: 14: 56.86$ & $-55: 52: 18.4$ & 1.86 & $\ldots$ & \\
KIC 2853093 & $19: 26: 19.00$ & $+38: 02: 09.0$ & $\ldots$ & $\ldots$ & \\
KIC 3547091 & $19: 28: 18.84$ & $+38: 37: 53.3$ & 8.74 & $\ldots$ & Contamination by nearby source at $W 3$ \\
KIC 3732821 & $19: 07: 40.11$ & $+38: 52: 20.0$ & 2.13 & $\ldots$ & Extended non-point source at $W 3$ \\
KIC 4545187 & $19: 04: 38.89$ & $+39: 40: 40.8$ & 0.83 & $\ldots$ & \\
KIC 6422367 & $18: 53: 53.07$ & $+41: 52: 23.6$ & 7.05 & 1.92 & Offset in $W 3$ \\
KIC 6665695 & $18: 48: 01.11$ & $+42: 10: 35.5$ & 0.49 & 2.25 & Extended non-point source at $W 4$ \\
KIC 6924203 & $18: 49: 19.88$ & $+42: 27: 49.8$ & 3.25 & $\ldots$ & Extended non-point source at $W 3$ \\
KIC 8414716 & $18: 57: 43.32$ & $+44: 28: 49.9$ & 1.93 & $\ldots$ & \\
KIC 9008220 & $19: 04: 36.48$ & $+45: 19: 57.2$ & 1.19 & $\ldots$ & \\
KIC 9214942 & $19: 21: 55.19$ & $+45: 36: 02.5$ & 1.19 & 2.05 & Extended non-point source at $W 4$ \\
\hline
\end{tabular}

Notes. These are no longer warm-excess candidates but are given here for reference to R12. ${ }^{(a)} \chi_{12}$ estimated by hand due to apparent excesses at $W 1$ and $W 2$ that yield standard R12 method inapplicable.

since they fulfilled the WISE excess and image inspection selection criteria, and the catalog of KOIs could grow with time to include any of the objects in the KIC catalog when more lightcurve data were analyzed jointly.

Table 2 gives the objects selected in R12 but with the updated $\chi_{\lambda}$ values and image inspection notes from the final WISE All-Sky Data Release. Half of the objects previously identified with the WISE PRC as having candidate warm excesses are discarded with the higher quality WISE ASC data, while the other half (namely KIC 3547091, KIC 3732821, KIC 6422367, KIC 6665695, KIC 6924203, and KIC 9214942) still fulfill our photometric excess criterion. In these objects, image inspection allowed us to rule them out as valid candidates for our study. Table 2 describes the issues detected during the image inspection. Some of these issues were also identified in the WISE ASC quality flags for bands $W 3$ and $W 4$, but only manual image inspection allowed us to identify them as bogus warm-excess objects.

\section{Observations, data reduction, and results}

Each of the 19 resulting targets were observed at 100 and $160 \mu \mathrm{m}$ using the PACS mini scan-map AOT, in the recommended two cross-scan directions (within the observing program OT2_dardila_2). The observations contained ten scan legs, three-minutes long and were repeated six times, in two concatenated AORs at angles 70 and 110 degrees with respect to the PACS detector. The total time per source was $3611 \mathrm{~s}$, achieving a $1 \sigma$ sensitivity of $\sim 1 \mathrm{mJy}$ at $100 \mu \mathrm{m}$ and $2-5 \mathrm{mJy}$ at $160 \mu \mathrm{m}$. The Herschel observation IDs (obsids) are given in Table 1 for each of the targets. Objects KIC 4143755 and KIC 7222086, both of which did not pass the $\chi_{\lambda}>2$ criteria in an a posteriori analysis with the WISE ASC, were also observed with obsid pairs 1342259362 and 1342259363 and 1342257738 and 1342257739 , respectively, within this program. Exactly the same analysis as for the targets satisfying all selection criteria was 
applied to them, and they were kept in the rest of the work as part of the control sample.

The images were processed with HIPE version 11.0 (Ott 2010) using the PACS point source i-pipe and the latest PACS photometer flux calibration (calTree version 48, see the PACS calibration documents ${ }^{5}$ for more information). The same results were also obtained with the same pipeline in HIPE 10.0 and with calTree version 45 . This is the default high-pass filtering pipeline with the standard 15- and 25-pixel high-pass filter widths for the 100 and $160 \mu \mathrm{m}$ images, respectively. To maximize point-source sensitivity at the center of the images, the user pipeline explicitly masks the source positions with a $20^{\prime \prime}$ mask before the final third pass of the high-pass filter takes place. The resulting pixel sizes are $2^{\prime \prime} \times 2^{\prime \prime}$ and $3^{\prime \prime} \times 3^{\prime \prime}$ for the 100 and $160 \mu \mathrm{m}$ images, respectively. All resulting images are shown in Fig. 1. The linear inverted grayscale goes from zero to $3 \sigma$, where the $\sigma$ is calculated as the standard deviation of the $50 \times 50$ central pixels in each image, where the coverage is best and relatively constant.

\subsection{Results}

No clear point sources were detected at any of the target positions. The spacecraft pointing of the observations of object KIC 3835670 was known to be affected by a reset of the spacecraft velocity vector (SVV) as identified during the quality control checks done at the Herschel Science Centre. This vector is used by the star tracker to compute the aberration correction of the coordinates of guide stars. To correct for these small offsets new pointing products were produced at the Herschel Science Centre with HCSS version 10.1 and applied to all affected observations from versions 11 onward (Sánchez-Portal et al. 2014). We also applied special checks to verify the pointing: using HIPE, we retrieved all PACS photometer observations in each of the days when our observations were taken and calculated the offsets between intended (nominal RA and DEC, as in the observations metadata) and achieved astrometry by fitting 2D Gaussians to any source found within $5^{\prime \prime} \times 5^{\prime \prime}$ around the intended central position of the images. This confirmed that the mean residual pointing offset on the days when our observations were taken was always smaller than $1.7^{\prime \prime}$. This rules out the possibility that large offsets would still be present in any of these images, hence that some of the nearby point sources could indeed be associated to our target stars.

The distances in arc seconds to the closest point-source emissions in the images within $50^{\prime \prime} \times 50^{\prime \prime}$ of the target positions were calculated by fitting 2D Gaussians on our images to the closest nearby point source and then calculating the distances between the 2D Gaussian peak position and the target positions. These values are given in Table 1 and range from 3 to $38^{\prime \prime}$. In particular, for a few targets, namely for HAT-P-28, KIC 3835670, and KIC 9703198, point sources are found close to the targets positions. For KIC 4918309 the source detection routine converged to a very nearby source as well, although probably has picked up a very low $\mathrm{S} / \mathrm{N}$ feature close to the target position. Doing blind aperture photometry with the recommended aperture of ten pixels in radius ( $2^{\prime \prime}$ for $100 \mu \mathrm{m}$ and $30^{\prime \prime}$ for $160 \mu \mathrm{m}$ ) at those positions could produce incorrect fluxes for these objects due to emission from unrelated sources falling into the apertures (see also, e.g., Matrà et al. 2012; Lestrade et al. 2012, for more research on confused far-IR fluxes). We therefore only computed upper limits to the fluxes at the positions of our sources. For

\footnotetext{
See PACS page at

http://www. cosmos. esa.int/web/herschel/home
}

this, we used the recommended method (implemented in the user pipeline script in HIPE 11.0) of estimating the RMS of 100 circular apertures of five pixels in radius $\left(10^{\prime \prime}\right.$ for $100 \mu \mathrm{m}$ and $15^{\prime \prime}$ and $160 \mu \mathrm{m})$ in a grid of $1.6^{\prime} \times 1.6^{\prime}$ for $100 \mu \mathrm{m}$ or $2.5^{\prime} \times 2.5^{\prime}$ for $160 \mu \mathrm{m}$, centered around the target positions. The flux upper limit values obtained are given in Table 1 and are in general consistent with the predictions from HSpot (v. 6.3.2) for the sensitivity of the PACS mini-scan AOT.

While most fields appear clean, few of them show extended emission at distances of $3^{\prime \prime}$ to $6^{\prime \prime}$ from the target positions. Extended far-IR background emission is found to be more common at $160 \mu \mathrm{m}$, even at such high galactic latitude fields as these. In particular for KIC 3835670, which has the strongest emission closest to the star's position, the extended emission has an extent of $29^{\prime \prime}$. Assuming main sequence stellar radius and luminosity for the star and comparing its $J$-band magnitude with the absolute magnitude of a star from its spectral type, we compute a photometric distance to this object of $\sim 600 \mathrm{pc}$, which then implies that the extended emission seen at the source position in the PACS maps would have a physical extension of $17400 \mathrm{AU}$ if it were associated to the star. This essentially rules out the possibility that this is a debris disk. There is a possibility that a $10 \mathrm{mJy}$ debris disk would be hidden among this bright background extended emission but it is impossible to prove that with the current data. Only higher resolution mid-IR imaging can help sort out whether the extended emission could be related to these sources, although it is highly unlikely given the projected distances and sizes of the background sources. This result then gives support to the thesis by Kennedy \& Wyatt (2012) that all these objects are likely due to chance alignment with either background galaxies or interstellar medium.

To constrain the possible dust content of the warm excess sources identified with WISE, we included the Herschel/PACS upper limit flux measurements at 100 and $160 \mu \mathrm{m}$ in the SEDs of the candidate mid-IR excess systems. The aim of this analysis is to quantify the relative constraints on the excess flux of these objects with the PACS far-IR wavelengths. To construct the SEDs we combined fluxes from the Tycho, SDSS, DENIS, 2MASS, and WISE for all targets and dereddened them with the Weingartner \& Draine (2001) interstellar extinction law using extinctions from the KIC or computing them for the transiting planet systems with the help of optical plus 2MASS fluxes. We compared the dereddened fluxes with NEXTGEN stellar atmospheres (Hauschildt et al. 1999) with the same spectral types of the stars as obtained from the Kepler Input Catalog or from http://exoplanets.org in the case of the transiting planet systems. The SEDs are shown in Fig. 2. They include the normalized SEDs of $\eta$ Crv (a prototypical warm excess debris disk, Matthews et al. 2010; Duchêne et al. 2014) and $\beta$ Pic (a prototypical massive debris disk, Vandenbussche et al. 2010). The 12 to $24 \mu \mathrm{m}$ excesses of these two prototypical sources are very different: while the 12 to $24 \mu \mathrm{m}$ slope of $\eta \mathrm{Crv}$ is rapidly declining, that of $\beta$ Pic rises. This indicates that only in the case of a bright massive debris disk, the rising 12 and $24 \mu \mathrm{m}$ excesses mark the Wien tail of a colder excess emission. Therefore in the common case of having only detected excesses at $12 \mu \mathrm{m}$, it is impossible to distinguish between the two models. Higher $\mathrm{S} / \mathrm{N}$ data around $24 \mu \mathrm{m}$ data for these objects is needed to achieve that.

On the other hand, while the large flux upper limits determined with the PACS data put weak constraints on the physical interpretation of the systems, these figures illustrate that conspicuous far-IR excesses like that in $\beta$ Pictoris (Vandenbussche et al. 2010) would have been detected with these observations in most of the targets if they were present in these objects. For the nearby 
B. Merín et al.: Herschel/PACS photometry of transiting-planet host stars with candidate warm debris disks
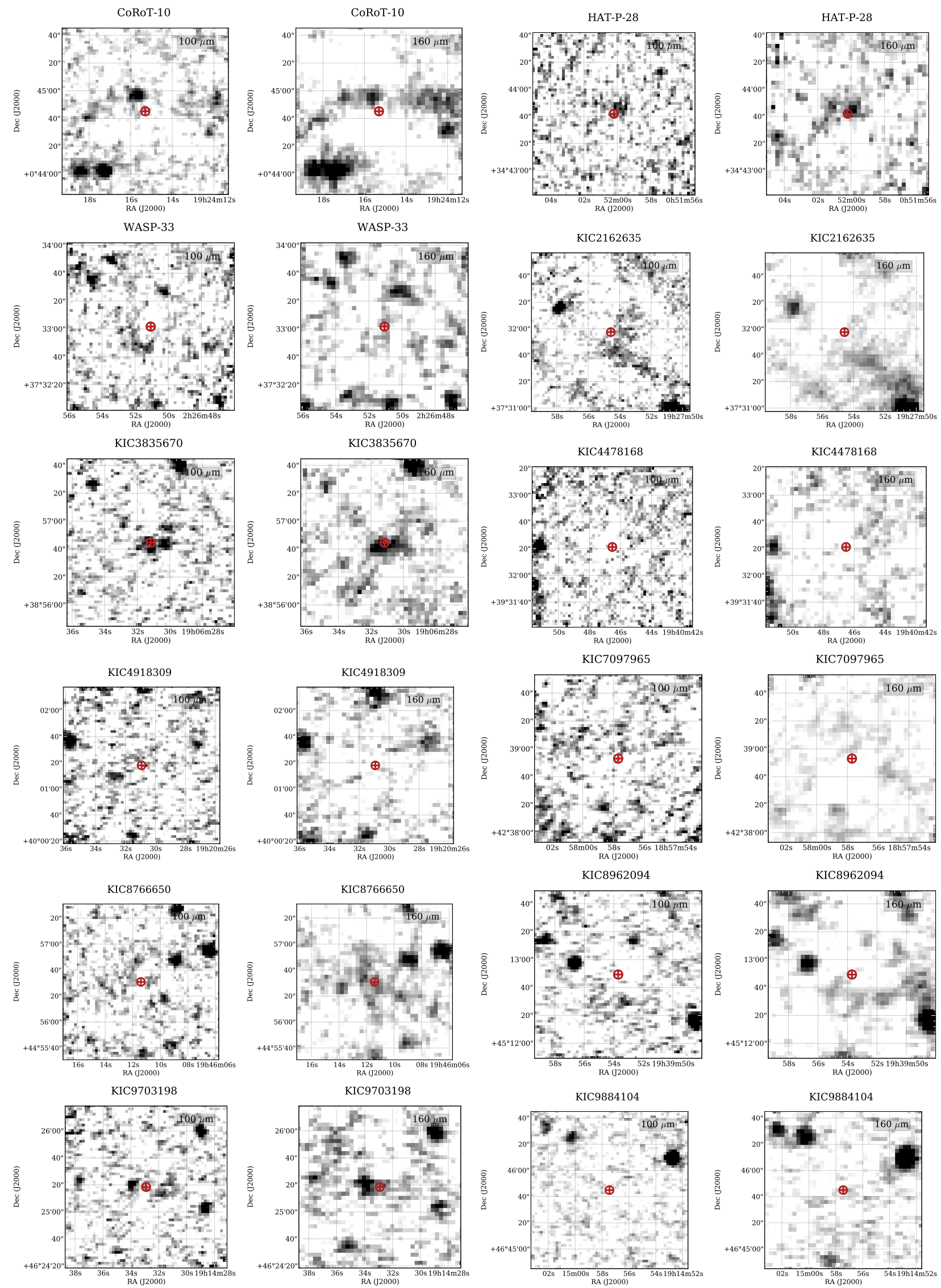

Fig. 1. Herschel/PACS images of the planet host and host candidates in the sample. The red target symbol indicate the nominal position of the target in the KIC catalog or in the Exoplanet Catalog. The linear inverted greyscale goes from zero to $3 \sigma$, where the $\sigma$ is calculated as the standard deviation of the $50 \times 50$ central pixels in each image. 

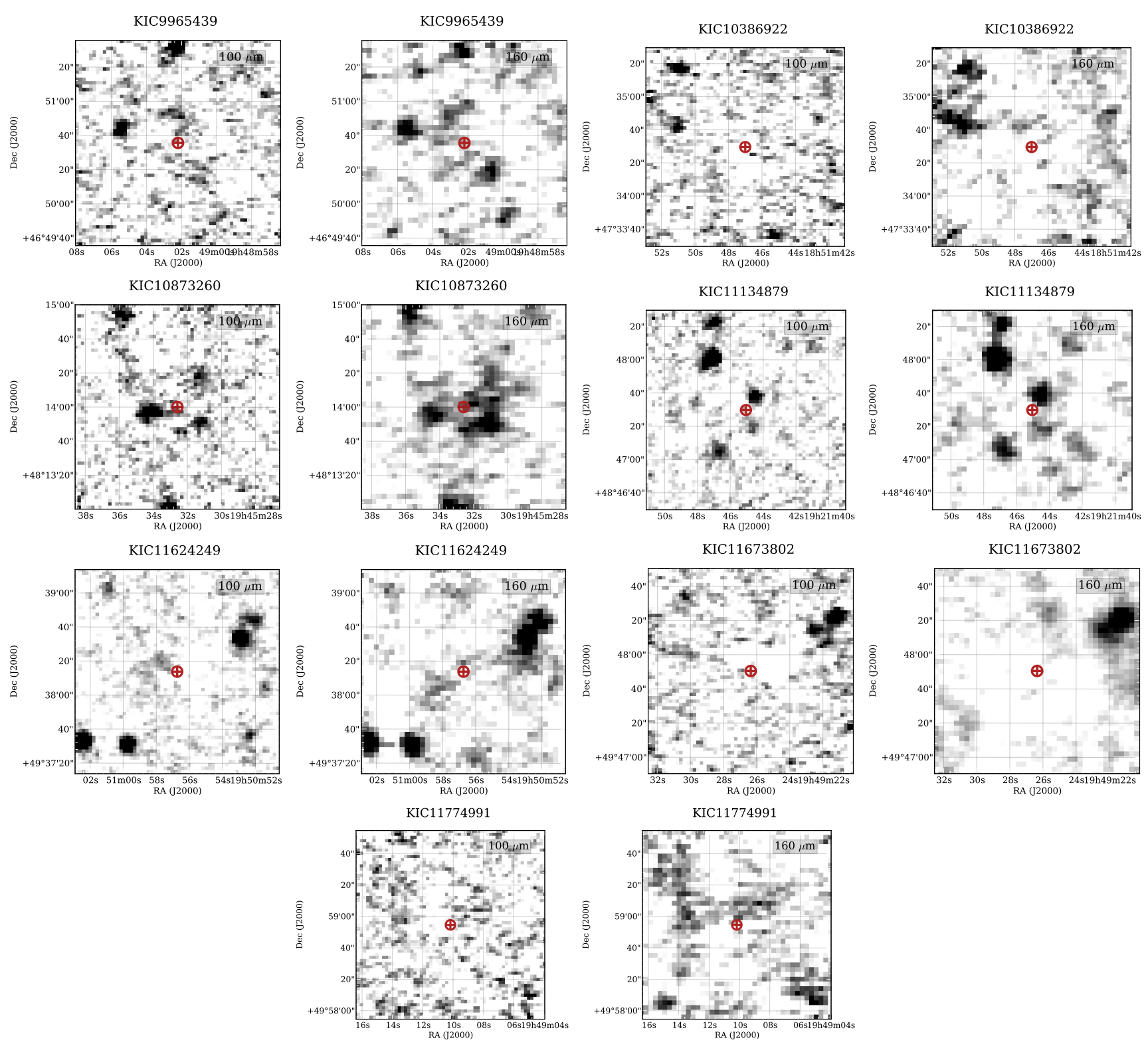

Fig. 1. continued.

exoplanet-host star WASP-33, the new PACS data allows us to also discard weaker excesses at $100 \mu \mathrm{m}$ and $160 \mu \mathrm{m}$ like those in $\eta$ Crv (Matthews et al. 2010; Duchêne et al. 2014). Therefore, our new PACS data allows us to rule out massive debris disks in most of these objects but cannot be used to rule out smaller excesses or warm-only debris disks.

\section{Discussion and conclusions}

Kennedy \& Wyatt (2012) argue that warm excess debris disk candidates identified with all-sky surveys like WISE do suffer from serious mid-IR background contamination and that the resulting numbers match the expected values of extragalactic counts suggesting that these warm-excess candidates might result from chance alignment with background galaxies. However, although reasonable, this is a statistical argument so it cannot rule out the presence of some such objects, such as in the case of the now famous $\eta$ Crv warm debris disk (Matthews et al. 2010; Duchêne et al. 2014).
An alternative direct check of this hypothesis, beyond highresolution mid-IR imaging of the candidates, is to search for farIR excesses on these same targets, which one would expect if the candidate excesses found in these objects were indeed associated to the objects and the disks were not extremely warm.

Having no result in this program confirms that the planethosting star WASP-33 does not have a debris disk comparable to that of $\eta \mathrm{Crv}$, that most other candidate mid-IR excess sources do not have a debris disk like that of $\beta$ Pic, and places a loose constraint on the presence of such rare warm disks around the Kepler planet-hosting candidates, given the sensitivity of the observations at the distance to the Kepler sample. However, this result and the nature of the emissions found at longer wavelengths support the hypothesis by Kennedy \& Wyatt (2012) that most of the WISE-identified mid-IR candidate excesses around these systems do indeed stem from chance alignment from either background IR-bright galaxies and/or interstellar emission.

Acknowledgements. This work has been possible thanks to the support from the ESAC Trainee Program, ESAC Space Science Faculty, and of the Herschel Science Centre. AR acknowledges support from the ESA SRE-OA Research 
B. Merín et al.: Herschel/PACS photometry of transiting-planet host stars with candidate warm debris disks
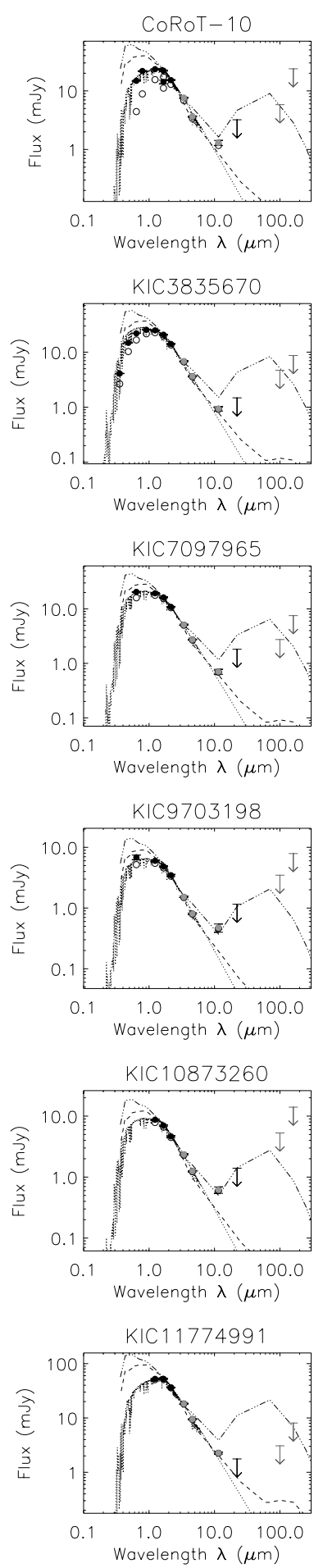
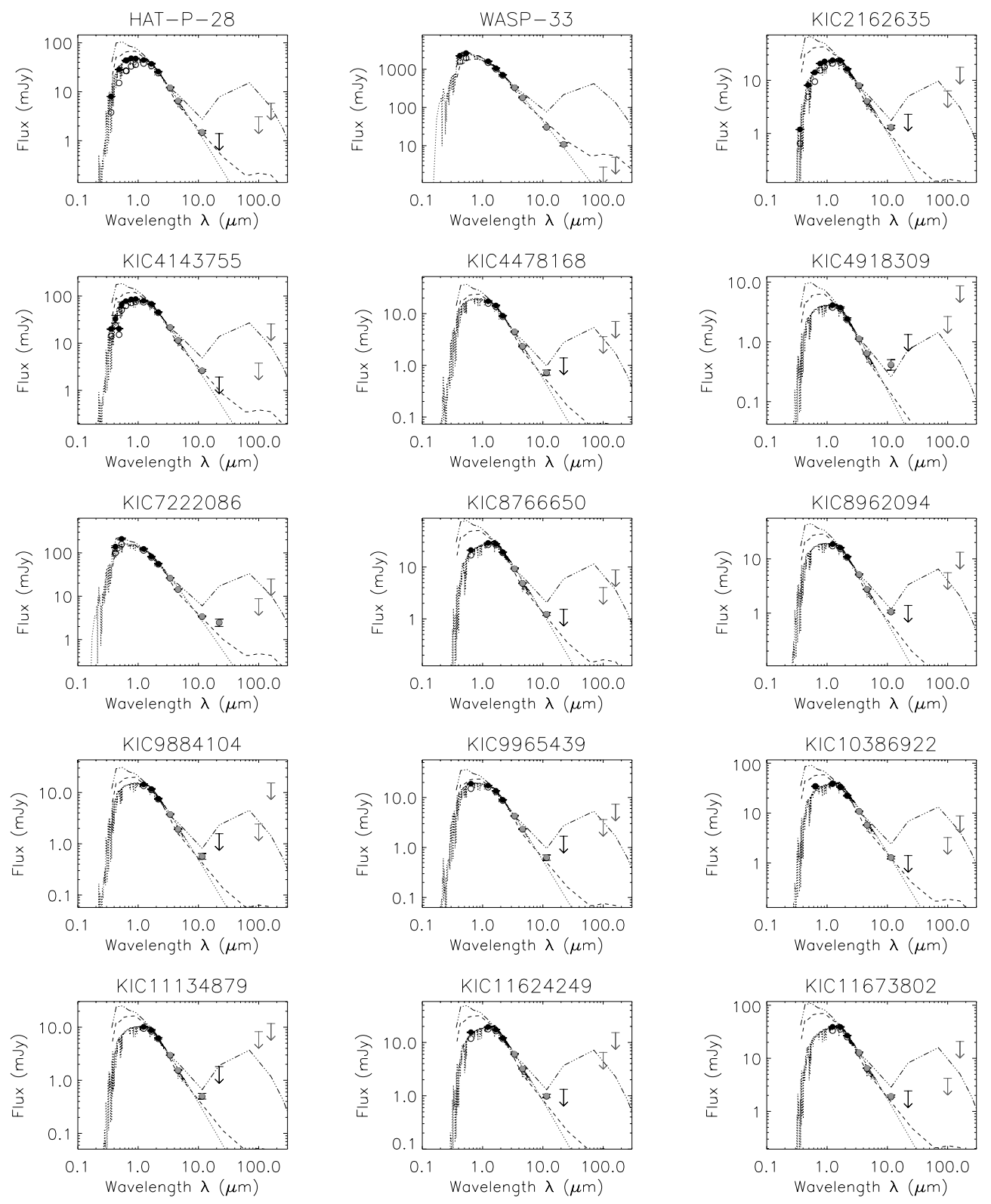

Fig. 2. Spectral energy distributions (SEDs) of planet hosts and planet host candidates. The dotted line represents the stellar photosphere model for the spectral type of the star scaled to the dereddened $J$-band flux. Open and solid dots are observed and dereddened fluxes, respectively. Symbols in gray are WISE fluxes. The two longest wavelength flux upper limits are the Herschel/PACS observations. For comparison, the SEDs of $\eta$ Crv (Duchêne et al. 2014) and $\beta$ Pic (Vandenbussche et al. 2010) have been normalized to the dereddened $J$-band flux and added to each plot denoted by dashed and dot-dashed lines, respectively.

Funding via contract SC 1300016149. Support for this work was provided by NASA through an award issued by JPL/Caltech. This publication is based on observations made by the Herschel Space Telescope and the PACS instrument. PACS was developed by a consortium of institutes led by MPE (Germany) and including UVIE (Austria); KU Leuven, CSL, IMEC (Belgium); CEA, LAM (France); MPIA (Germany); INAF-IFSI, OAA, OAP, OAT, LENS, SISSA (Italy); IAC (Spain). This development has been supported by the funding agencies BMVIT (Austria), ESA-PRODEX (Belgium), CEA/CNES
(France), DLR (Germany), ASI, INAF (Italy), and CICYT/MCYT (Spain). HCSS, HSpot and HIPE are joint developments by the Herschel Science Ground Segment Consortium, consisting of ESA, the NASA Herschel Science Center, and the HIFI, PACS and SPIRE consortia. This publication is also based on observations made with the Kepler Spacecraft. Funding for this mission is provided by National Aeronautics and Space Administration's Science Mission Directorate (NASA). This study also makes use of data products from Herschel Space Observatory and the Wide-field Infrared Survey Explorer, 
a joint project of the University of California, Los Angeles, and the Jet Propulsion Laboratory (JPL)/California Institute of Technology (Caltech); the NASA Infrared Processing and Analysis Center (IPAC) Science Archive and the NASA/IPAC/NExScI Star and Exoplanet Database, operated by JPL, Caltech, and funded by NASA; the SIMBAD database and the Vizier service, operated at the Centre de Donnes astronomiques de Strasbourg, France; the data products from the Two Micron All Sky Survey (2MASS), a joint project of the University of Massachusetts and IPAC at Caltech, funded by NASA and the National Science Foundation; the Multimission Archive at the Space Telescope Science Institute (MAST). STScI is operated by the Association of Universities for Research in Astronomy, Inc., under NASA contract NAS5-26555. Support for MAST for non-HST data is provided by the NASA Office of Space Science via grant NNX09AF08G and by other grants and contracts.

\section{References}

Absil, O., di Folco, E., Mérand, A., et al. 2006, A\&A, 452, 237 Absil, O., Defrère, D., Coudé du Foresto, V., et al. 2013, A\&A, 555, A104 Adams, E. R., Dupree, A. K., Kulesa, C., \& McCarthy, D. 2013, AJ, 146, 9 Aumann, H. H., Beichman, C. A., Gillett, F. C., et al. 1984, ApJ, 278, L23 Batalha, N. M., Borucki, W. J., Koch, D. G., et al. 2010, ApJ, 713, L109

Duchêne, G., Arriaga, P., Wyatt, M., et al. 2014, ApJ, 784, 148

Eiroa, C., Marshall, J. P., Mora, A., et al. 2013, A\&A, 555, A11

Hauschildt, P. H., Allard, F., \& Baron, E. 1999, ApJ, 512, 377

Kennedy, G. M., \& Wyatt, M. C. 2012, MNRAS, 426, 91

Latham, D. W., Brown, T. M., Monet, D. G., et al. 2005, in BAAS, 37, 1340

Lawler, S. M., \& Gladman, B. 2012, ApJ, 752, 53
Lawler, S. M., Beichman, C. A., Bryden, G., et al. 2009, ApJ, 705, 89 Lestrade, J.-F., Matthews, B. C., Sibthorpe, B., et al. 2012, A\&A, 548, A86 Lillo-Box, J., Barrado, D., \& Bouy, H. 2014, A\&A, 566, A103

Mann, A. W., Gaidos, E., Lépine, S., \& Hilton, E. J. 2012, ApJ, 753, 90 Mannings, V., \& Barlow, M. J. 1998, ApJ, 497, 330

Matrà, L., Merín, B., Alves de Oliveira, C., et al. 2012, A\&A, 548, A111

Matthews, B. C., Sibthorpe, B., Kennedy, G., et al. 2010, A\&A, 518, L135 Melis, C., Zuckerman, B., Rhee, J. H., \& Song, I. 2010, ApJ, 717, L57

Moro-Martín, A. 2008, in eds. Y.-S. Sun, S. Ferraz-Mello, \& J.-L. Zhou, IAU Symp., 249, 347

Morton, T. D., \& Johnson, J. A. 2011, ApJ, 738, 170

Ott, S. 2010, in Astronomical Data Analysis Software and Systems XIX, eds. Y. Mizumoto, K.-I. Morita, \& M. Ohishi, ASP Conf. Ser., 434, 139

Oudmaijer, R. D., van der Veen, W. E. C. J., Waters, L. B. F. M., et al. 1992, A\&AS, 96, 625

Pilbratt, G. L., Riedinger, J. R., Passvogel, T., et al. 2010, A\&A, 518, L1

Poddaný, S., Brát, L., \& Pejcha, O. 2010, New Astron., 15, 297

Poglitsch, A., Waelkens, C., Geis, N., et al. 2010, A\&A, 518, L2

Ribas, Á., Merín, B., Ardila, D. R., \& Bouy, H. 2012, A\&A, 541, A38

Sánchez-Portal, M., Marston, A., Altieri, B., et al. 2014, Exp. Astron., 37, 453

Siegler, N., Muzerolle, J., Young, E. T., et al. 2007, ApJ, 654, 580

Skrutskie, M. F., Cutri, R. M., Stiening, R., et al. 2006, AJ, 131, 1163

Su, K. Y. L., Rieke, G. H., Malhotra, R., et al. 2013, ApJ, 763, 118

Trilling, D. E., Bryden, G., Beichman, C. A., et al. 2008, ApJ, 674, 1086

Vandenbussche, B., Sibthorpe, B., Acke, B., et al. 2010, A\&A, 518, L133

Weingartner, J. C., \& Draine, B. T. 2001, ApJ, 548, 296

Wyatt, M. C. 2008, ARA\&A, 46, 339

Wyatt, M. C., Smith, R., Greaves, J. S., et al. 2007, ApJ, 658, 569 